\title{
Histological and immunohistochemical parameters of the skin under the influence of damaging and stimulating cosmetic procedures
}

\begin{abstract}
The article presents the results of the research of the content of collagen types I and III in the skin, the growth factors of FGF2, EGF, VEGF before and after exposure to the skin of an erbium laser as monotherapy and under complex laser and biorevitalization. A comparative characteristic of these changes was made; conclusions were drawn about the effectiveness of the therapy
\end{abstract}

Keywords: collagen, growth factors, skin rejuvenation, erbium laser, biorevitalization
Volume 2 Issue 2 - 2018

\author{
Ziganshin O, Kudrevich Y, Kuznetsova E, \\ Sichugov G, Zayats T, Emelina A \\ South Ural State Medical University, Russia
}

Correspondence: Ziganshin O, South Ural State Medica University 454048, Chelyabinsk, Russia, Tel 89630860003 , Email ziganshin_oleg@mail.ru

Received: January 31, 2018 | Published: March 27, 2018

\section{Introduction}

The relevance of the issue of the effectiveness, safety and effectiveness of cosmetic procedures is very high today. Over the past 10-15years, a large number of methods have appeared that have a stimulating effect on the skin, leading to its rejuvenation, tonification, improvement of skin quality, etc. ${ }^{1}$ Often, the methods used for this purpose have a damaging effect, for example, the action of an ablative erbium laser. The mechanisms of the erbium laser action are reduced to damage to the epidermis by heating the water in the cells and the intercellular space and evaporating it, thereby breaking the integrity of the epidermis. The depth of damage to the epidermis depends on the parameters of the laser radiation and varies from damage to the stratum corneum to damaging the prickly layer of the epidermis. After such damage universal inflammation processes and mechanisms of skin regeneration are triggered, the epidermis is restored, young cells of the epidermis are formed, also occurs stimulation of the dermis fibroblasts, an increase in the number of collagen fibers, young cells of the epidermis are formed, the stimulation of the dermal fibroblasts, an increase in the number of collagen fibers, the formation of new microcirculatory vessels. ${ }^{2-5}$

In addition, the procedure of skin biorevitalization is very popular, which consists in introducing into the skin of native hyaluronic acid a certain molecular mass and concentration. ${ }^{6}$ Hyaluronic acid, located in the dermis, retains water in the intercellular space, gives the skin turgor, acting on the receptors of CD44 fibroblasts and keratinocytes, increases the activity of these cells, increases the synthesis of components of the intercellular matrix, including collagen, elastin and, to a greater extent hyaluronic acid.

CD44 receptors are markers of epidermal growth and normal differentiation of epidermal cells. The receptor for hyaluronanmediated motility (RHAMM) is also involved in the interaction of cells with external hyaluronic acid. RHAMM provides an increase in the quality of epithelization in a medium rich in hyaluronic acid, the migration of cells into the inflammatory focus in the dermis, where the decomposition of hyaluronic acid is activated. Vascular endothelial cells have RHAMM. Their interaction with hyaluronic acid causes the activation of vascular endothelial cells, which leads to the growth of new capillaries. Hyaluronic acid fulfills one more function - it protects DNA from oxidants. ${ }^{7}$ This mechanism is carried out by trapping iron ions and suppressing, therefore, the Fenton reaction, which results in the formation of secondary oxidants. It is also like an antioxidant that directly removes the primary and secondary reactive oxygen intermediates in the decomposition of the acid itself. ${ }^{8-11}$

In general, the effects of various cosmetic procedures, such as discoloration, smoothness of the relief, lifting of different areas of the face, are evaluated subjectively by doctor and patients. Fixation and comparison of results before and after procedures is carried out by photographing the patient. There are very few literary sources in which data of evidence-based medicine are given with respect to the amount of collagen, elastin, hyaluronic acid, the degree of vascularization of the dermis after various effects, in addition, the data are scattered. There is little data on a comprehensive assessment of the skin condition before and after the effects aimed at improving the quality of the skin.

The purpose of our work is to assess the histological and immunohistochemical parameters of the skin before and after the action of ablative erbium laser in monotherapy and in combination of this effect with biorevitalization., as well as assess the degree of skin changes with monotherapy and combined therapy with two methods, to identify the safest and most effective methods of affecting the quality of the skin.

The research involved 24patients, women from 40 to 60years old, whose average age was $48.4 \pm 5.14$ years. This age group was chosen not accidentally, age changes of the skin are already expressed in this age period but the regenerative potential of the organism is quite strong.

\section{Criteria for inclusion in the study}

Age: 40-60years, signed informed consent for participation in the research, understanding of the procedure of the study and readiness 
to follow the recommendations of the researcher during a 2 month research, the presence of signs of chronostenia, readiness for voluntary punch biopsy next to the treatment area (skin above the mastoid process).

Exclusion criteria: planning for any other cosmetic procedures in the face area during the research, any facial cosmetic procedures during the last 6 months before the start of the research, infectious diseases in the area of drug administration, the use of any absorbable fillers, botulinum toxin type $\mathrm{A}$ in the field of treatment for $12 \mathrm{months}$ before the start of the research, pregnancy, lactation.

\section{Materials and methods}

All patients were divided into two groups of 12people. The first group of patients underwent laser treatment of the face and the right occipital region in the projection of the mastoid process. It was used an erbium laser with a wavelength of $2940 \mathrm{~nm}$, the wave energy was $96 \mathrm{~J} / \mathrm{cm}^{2}$. Treatment was carried out once after anesthesia of the skin with a local anesthetic containing lidocaine.

The second group of patients underwent a combined procedure involving a single biorevitalization procedure with a combined preparation containing hyaluronic acid, whose molecular weight was $3 \mathrm{KDa}$, in a concentration of $10 \mathrm{mg} / \mathrm{ml}(1 \%)$ and trehalose at a concentration of $0.02 \%$, the skin of the face and the right occipital area was treated. After biorevitalization, the skin of the face and the behind-ear region was immediately treated with an erbium laser. Anesthesia was also local.

All patients had a fragment of skin in the right occipital region in the projection of the mastoid process to the procedure and 2 months after the procedures. The fence was performed by the method of punch biopsy, the diameter of the dermopanche was $3 \mathrm{~mm}$, and the column of the skin included a fragment of the epidermis and dermis.

In skin samples using immunohistochemical methods with the help of monoclonal mouse antibodies, the following parameters were determined: bulk density of type I and III collagen, growth factors FGF2, EGF, VEGF. The biological activity of FGF is diverse. It is a mitogen for various cells of neuroectodermal and mesenchymal origin, a potential mitogen and a stimulator of neoangiogenesis, support and stimulates the differentiation of cells of various neuronal types in vivo and in vitro.
VEGF is a potential mitogen for vascular epithelial cells. It exerts a strong influence on the permeability of blood vessels, it is a powerful angiogenic protein, participates in the processes of neovascularization. EGF is a strong mitogen for various cells of endodermal, ectodermal and mesodermal origin. The main place of EGF synthesis is the salivary glands. EGF monitors and stimulates the proliferation of epidermal and epithelial cells, including fibroblasts, renal epithelium, glial cells and thyroid cells in vitro. EGF itself and in combination with other cytokines is the most important factor mediating the processes of wound healing and angiogenesis. Collagen I, III and V are fibrillar collagens. This is the bulk of the collagen in the skin, which is a fiber woven from bundles of fibrils. About $95 \%$ of all collagen in the human dermis are collagen types I and III, which form very strong fibrils. Moreover, collagen type I - the main "adult" and "strong" collagen, and type III collagen is the collagen of the embryonic dermis, it is very young collagen.

$\mathrm{V}$ type collagen is combined in fibrils with collagen of the first and third types, and participates in the regulation of the diameter of fibrils.

\section{Results and discussion}

When comparing the amount of collagens I and III types, growth factors in patients of both groups that received cosmetology procedures, the content of these indicators after the procedure is increased. So in the group of patients who received the procedure of fractional photothermolysis, the volume density of type I collagen from the initial figure before the procedure of $13.58 \pm 1.56$ increased to $17.44 \pm 1.07$, the increase was $23 \%$, the changes were significant $(p<0.05)$. In the group of patients who received a combined effect on the skin, including fractional photothermolysis and biorevitalization, this figure was before the procedure $15.89 \pm 0.81$, after the procedure there was an increase in this indicator to $16.26 \pm 0.63$, an increase is unreliable.

There were changes in the bulk density of type III collagen: in the first group of patients before the procedure the bulk density was $19.54 \pm 1.52$, after the procedure it increased and became equal to $20.63 \pm 1.08$, the increase was not significant. In the second group before the procedure it was $18.63 \pm 1.79$, after the procedure it increased by $10 \%$ and amounted to $20.67 \pm 2.12(\mathrm{p}<0.05)$. The data are presented in Table 1.

Table I Comparative indices of collagen content of types I and III in patients who received various cosmetic procedures

\begin{tabular}{llll}
\hline & & Collagen type I, am\% & Collagen type III, am\% \\
\hline \multirow{2}{*}{ I group "laser" } & Before the procedure & $13,58 \pm I, 56$ & $19,54 \pm I, 52$ \\
& After the procedure & $\mid 1,44 \pm I, 07 *$ & $20,63 \pm I, 08$ \\
2 group "Laser + revitalization" & Before the procedure & $15,89 \pm 0,81$ & $18,63 \pm I, 79$ \\
& After the procedure & $16,26 \pm 0,63$ & $20,67 \pm 2, I 2 *$ \\
\hline
\end{tabular}

$* \mathrm{p}<0,05$.

When comparing the growth factors in different groups, we obtained the following data: in the first group of patients the bulk density of fibroblast growth factor FGF2 before the procedure was $1.85 \pm 0.87,2$ months after the procedure of fractional photothermolysis it increased to $2.0 \pm 0.86$, the changes were unreliable. In the second group of patients this index was $1.22 \pm 0.68$, after the procedure it significantly increased and became $2.5 \pm 0.33(\mathrm{p}<0.5)$.
The rest of the growth factors in each of the groups also increased after the procedure, the increase were $17-23 \%$. So the epidermal growth factor (EGF) in the group of patients who received the procedure of laser exposure before the procedure was $9.72 \pm 1.49$, after the procedure its value was $14.43 \pm 2.32(\mathrm{p}<0.5)$. Endothelial growth factor (VEGF) in the same group before the procedure was $1.89 \pm 0.66$, after the procedure- $2.3 \pm 0.43(\mathrm{p}<0.5)$. In the second group that 
received a complex effect, EGF before the procedure was $7.66 \pm 2.03$, after the procedure its value increased to $8.87 \pm 1.46,(\mathrm{p}<0.5)$, VEGF before the procedure was $1.54 \pm 0.12$, two months after the procedure it was already $1.84 \pm 0.11,(\mathrm{p}<0.5)$. The data are presented in Table 2 .

Table 2 Comparative characteristics of growth factors in patients who received different cosmetic procedures

\begin{tabular}{|c|c|c|c|c|}
\hline & & FGF2, am\% & EGF, am\% & VEGF, am\% \\
\hline \multirow{2}{*}{ I group "laser" } & Before the procedure & $\mathrm{I}, 85 \pm 0,87$ & $9,72 \pm 1,49$ & $1,89 \pm 0,66$ \\
\hline & After the procedure & $2,0 \pm 0,86$ & $14,43 \pm 2,32 *$ & $2,3 \pm 0,43 *$ \\
\hline \multirow{2}{*}{2 group "Laser + revitalization" } & Before the procedure & $1,22 \pm 0,68$ & $7,66 \pm 2,03$ & $1,54 \pm 0,12$ \\
\hline & After the procedure & $2,5 \pm 0,33 *$ & $8,87 \pm 1,46^{*}$ & $\mathrm{I}, 84 \pm 0, \mathrm{I} \mid *$ \\
\hline
\end{tabular}

$* \mathrm{p}<0,05$.

\section{Conclusion}

Based on the data we can be concluding that cosmetic procedures stimulate neocollagenogenesis, contribute to the improvement of skin quality, its density. It can be confirmed by an increase in the number of collagens in the skin after the procedure compared with the same indicators before the procedure. The same is confirmed by data on the change in the number of growth factors that have a direct effect on the receptors of cells and, penetrating into the nucleus of cells, are direct mitogens. Based on the data on the increase in the amount of VEGF, it can be assumed that vascularization of the dermis will also be intensified, which will lead to increased skin trophism, enhanced metabolic processes, optimization and enhancement of regenerative processes, as well as immunological skin protection processes. Given that the greatest increase occurred in FGF precisely with the complex effect of laser and biorevitalization, it can be assumed that the most effective for the better, the skin parameters will change precisely with the a combined effect that involves damage to the epidermis by fractional photothermolysis and stimulation of skin regeneration processes, neocollagenogenesis, hydration of the dermis by introducing into the dermis native hyaluronic acid in combination with trehalose.

\section{Acknowledgements}

None.

\section{Conflicts of interest}

The authors declared that there are no conflicts of interest.

\section{References}

1. Nefedieva Yu V. Mesotherapy: drugs, techniques, side effects and complications. Nefedeva Yu V, Ziganshin OR, Kamyshina MA, editors. A manual for residents. Chelyabinsk: Russia; 2016.
2. Markelova EV, Yutskovskaya Ya A, Yutskovsky AD, et al. Inflammation as an integral part of injectable cosmetic manipulations. South Urals Medical Journal. 2015;1:31-37.

3. Jie $\mathrm{C}$, Wang $\mathrm{T}$, Yinfei $\mathrm{P}$, et al. Influence on proliferation and adhesion of human gingival fibroblasts from different titanium surface decontamination treatments: An in vitro study. Arch Oral Biol. 2017;87:204-210.

4. Li D, Lin SB, Cheng B. Complications and post treatment care following invasive laser skin resurfacing: A review. $J$ Cosmet Laser Ther. 2017;19:1-11.

5. Shen J, Lu XG, Jin JJ, et al. Combination of a 2940nm Er:YAG laser with recombinant bovine basic fibroblast growth factor (rb-bFGF) and lightemitting diode-red light (LED-RL) for the treatment of striae alba: A pilot study. J Cosmet Dermatol. 2017;17(2):176-183.

6. Kudrevich Yu V, Sychugov GV, Ziganshin OP. Anatomico-physiological changes in the skin during the procedure of biorevitalization. South Ural Medical Journal. 2012;1:38-41.

7. Ziganshina TA. Influence of antioxidants and high-molecular unstabilized hyaluronic acid on the properties of fillers and biorevitalizanolates. South Urals Medical Journal. 2014;1:41-43.

8. Bazarny VV. The immune system of the skin. Mesotherapy. 2011;2(14):28-36.

9. Bazarny VV, Valamina IE, Tikhonina EA, et al. Immunomodulation with ribomunyl of reparative processes in the skin of rats. Bull Exp Biol Med. 2007;143(6):660-662.

10. Gubanova E, Chaykovskaya E. Intradermal injections of hyaluronic acid: possible mechanisms for the formation of clinical effects. Injection methods in cosmetology. 2009;1:20-30.

11. Kudrevich Yu V, Sychugov GV, Ziganshin OR, et al. Changes in the structural parameters of the skin under the influence of biorevitalization. Ural Medical Journal. 2016;3(136):113-115. 